\title{
SOME ASPECTS OF CALCULATION OF JOINT OPERATION OF SEWAGE SLUDGE PUMPS AND PIPELINE NETWORKS
}

Viktor Andreevich Morozov*

Southwest State University, Kursk, Russia

Aleksandr Viktorovich Morozov

Southwest State University, Kursk, Russia

Elena Nikolaevna Morozova

Southwest State University, Kursk, Russia

The article considers the issues of joint operation of centrifugal pumps and networks for sewage sludge (SS) the flow of which is described by the Bingham plastic model. The authors present the results of the experimental studies of SS pumps operation and a formula for working out pump performance parameters for SS with different concentrations. To determine the design point of the joint operation of pumps and networks we used the dependence of hydraulic pressure losses in the pipeline by means of generalized Reynolds number taking into account SS viscoplastic properties. The results of experimental and design data for centrifugal SS pump performance parameters are given.

Key words: Sewage sludge, Pumps, Pipelines, Characteristics, Hydraulic pressure, Friction losses, Head, Discharge

\section{INTRODUCTION}

Pumping of natural and waste waters by centrifugal pumps consumes up to 150 billion $\mathrm{kWh}$ of electricity in the Russian Federation. Russian water and sewage plants' electricity costs are about $80 \%$ of their total expenditures. The calculation of the joint operation of pumping stations and networks is directly related to energy saving and performance reliability improving. Wide application of pumps for SS pumping sets an important practical task - determination of pump performance parameters for such fluids.

There are research data on the analysis of such works and recommendations for the calculation of the operation of pumps and networks only for the design conditions, which does not allow considering the work as a whole, since there is no visual clarity and the parameters for the joint operation of pumps and networks are not determined.

\section{A TECHNIQUE FOR CALCULATION OF JOINT OPERATION OF PUMPS AND NETWORKS}

The analysis of the joint operation of pumps and networks makes it possible to predict pump performance parameters for fluids, the flow of which is described by a model other than a water model. Correlation of SS pump performance parameters and pipeline network performance parameters determines the design point of the joint operation of pumps and networks [01].

To calculate the joint operation of pumps and networks, we use the Bingham plastic model which is successfully used to calculate the tasks of transportation of such hydraulic mixtures as peat mass, chalk and water-coal slurries, bog muck, SS, slurries; in this case the dimensionless similarity criterion, i.e. Reynolds number, for a round pipe is used; it takes into account the viscoplastic properties of pumped fluids [01, 04]:

$$
\begin{aligned}
& \operatorname{Re}^{*}=\frac{\mathrm{Re}}{1+0,3 \cdot \mathrm{Se}} \\
= & \frac{\rho V r}{\mu}, S e=\frac{\tau_{m} r}{\mu V} ;
\end{aligned}
$$


where;

$-p$ is the fluid density,

- $V$ is the flow rate in the pipe,

$-r$ is the radius of the pipe,

- $T$ is the yield value,

$-\mu$ is the coefficient of dynamic viscosity.

We obtained the operating pump performance parameters for sewage sludge (SS) of different concentration with the simultaneous determination of SS rheological properties at the test setup using the method of determining hydraulic losses in a section of the circular pipe.

As a result of the research, the dependence of hydraulic losses in pumps on the operating condition and SS rheological properties was obtained [01, 03]:

$$
\frac{1-\eta_{a c}}{1-\eta_{a}}=\left(\frac{\lambda_{c}}{\lambda}\right)^{\frac{Q}{Q_{p}}-0,5}
$$

where $\mu_{a c}$ and $\mu_{a}$ are the pump hydraulic efficiency for SS and water, respectively;

$\lambda_{c}$ and $\lambda$ are the hydraulic resistance coefficients for SS and water, respectively;

$Q$ is the current pump flow rate;

$Q_{p}$ is the design pump flow rate.

To generalize the data in formula (2), we used the results of the other authors' works for various fluids described by the Bingham plastic model.

In this case, the SS actual pump head is determined by:

$$
H_{c}=H_{m}\left[\left(\frac{1-\eta_{z}}{\left(\lambda_{c / \lambda}\right)^{\frac{Q}{Q_{p}}-0,5}}\right)-1\right]
$$

where $H_{c}$ is the SS actual pumping station head.

Formula (2) allows determining actual pump head for different operating conditions and build a SS head-discharge characteristic of the pump.

It is reasonable to determine the pump heads for three flow conditions (underloaded, design and overloaded conditions) to build the operating characteristic of the SS pump.

The change in the SS pump power consumption occurs solely due to the change in the pump mechanical efficiency in which the power of disk friction is of significant importance. Power losses in bearings and seals do not depend on the properties of the pumped fluid; therefore, having determined the power of disk friction in SS pump and having data concerning the power consumed by the pump for water, it is possible to construct the SS power consumption characteristic.

To calculate power consumption, it is necessary to determine the power of disk friction by the following formula:

$$
\mathrm{N}=\frac{1}{4} \rho \omega^{3} \mathrm{r}^{5} \mathrm{C}
$$

where $\mathrm{c}$ is the coefficient of disk friction, $\omega$ is the angular velocity,

$r$ is the radius of the pump impeller disc.

To calculate the coefficient of disk friction we obtained the calculation formulas for SS laminar flow condition. The first one is for the case of closed boundary layers (a small gap between the impeller disc and pump housing) [01,03]:

$$
C=\frac{2 \pi r}{\delta R e^{*}}
$$

The second one is for the case of separate boundary layers between the impeller disc and pump housing $[01,03]$ :

$$
C=\frac{2,82}{\sqrt{R_{e}^{*}}}
$$

where

$$
R_{e}^{*}=\frac{R_{e}}{1+\frac{4}{3} S e}, R e=\frac{\rho V r}{\mu}, S e=\frac{\tau_{m} \delta}{\mu V}
$$

$V$ is the circumferential velocity at the impeller inlet and outlet, $\sigma$ is the boundary layer thickness, $T$ is the yield value, $\mu$ is the coefficient of dynamic viscosity.

The formulas for determining the coefficient of disk friction were obtained by calculation and confirmed experimentally using the facility for studying disk friction [01].

Since the power consumed by the pump changes only depending on the power of disk friction, the SS pump power consumption, accurate to the amount of leakage in the front seal, can be 
built in parallel with the pump power consumption for water for the design condition.

The Darcy formula was used to build SS transportation network characteristics; in this formula, the coefficient of hydraulic friction is determined according to the dependence:

$$
\lambda=\frac{64}{R_{e}^{*}}
$$

where

$$
\begin{aligned}
& R_{\theta}^{*}=\frac{R_{\theta}}{1+0,3 S_{\theta}} ; R_{e}=\frac{V d}{\mu} \\
& S e=\frac{\tau_{m} r}{\mu V}
\end{aligned}
$$

The required head for SS supply to the treatment plant is determined by the dependence:

$$
H=H_{e}+h_{6}+h_{H}+h_{C}
$$

where $H_{a}$ is the geometric head;

$h_{b}$ is the hydraulic loss of suction head;

$h_{H}$ is the hydraulic head loss at discharge;

$h_{c}$ is the hydraulic head loss within station communications.

Having determined the hydraulic losses in the pipeline and having set a series of values of $Q_{1}$, $Q_{2}, \ldots, Q_{i}$, we build a characteristic curve of the network.

\section{RESULTS OF EXPERIMENTAL STUDIES}

The operating mode of the SS pumping station was determined by superposing network characteristics on pump characteristics. Graph analysis of the joint operation of sewage pumping stations and networks makes it possible to calculate the performance points for pumping station operation.

Figure 1 shows the results of pilot tests of SD $160 / 45$ SS pump.
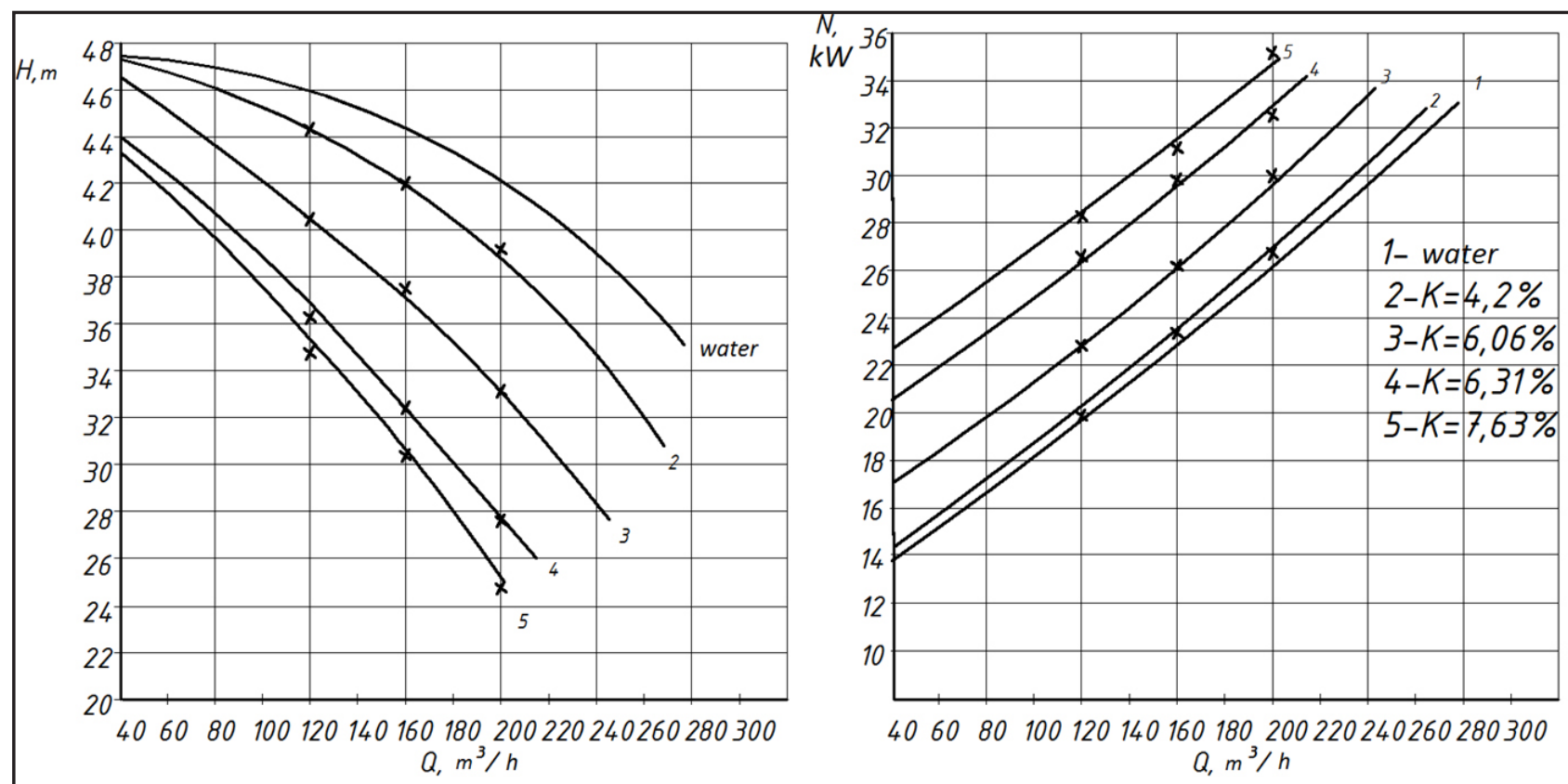

Figure 1: Comparison of the experimental and design characteristics of CD 160/45 SS pump: - - experimental test data; $x$ - design characteristics

As it can be seen from Figure 1, the experimental data of SD 160/45 pump tests correspond to the results of the design pump characteristics, obtained by the technique using dependencies $(2,3,5,6)$. The maximum error is $8 \%$.

\section{CONCLUSION}

The proposed dependences $(2,3,5,6)$ make it possible to recalculate pump performance parameters worked out for water to SS pump parameters. 
The use of the Bingham plastic model allows us to recommend this technique for recalculation of the parameters for other fluids, the flow of which is described by this model.

Graph-analytical method for calculation of the joint operation of SS pumps and networks determines the parameters of pump head, flow rate and power consumption for this network.

\section{REFERENCES}

1) Morozov A. V. Calculation of the Sewage Pumping Stations parameters [Text] / A. V. Morozov, V. A. Morozov // Industrial and Civil Construction Engineering. - 2009. - No 5. - P. 42-43.

2) Morozova E. N. Design and Construction Improvement of Sewage Pumping Stations Reliability as a Factor of Improving Cities Ecological Safety. E. N. Morozova, V. A. Morozov // Designing and Construction: a collection of abstracts of scientific and practical conference reports. - Kursk, 2015. - P. 39-40.

3) Scherbakov V. I. Prediction of Performance Parameters of Sewage Sludge Centrifugal Pumps [Text] / V. I. Shcherbakov, A. V. Morozov // "Yakovlev Readings". X Scientific and Technical Conference. Collection of reports. - Moscow: ASV Publishing House, 2015. - P. 61-65.

4) Sherstyuk, A. N. Calculation of the Centrifugal Pumps Performance Parameters for viscoplastic fluids transportation / A. N. Sherstyuk, V. A. Morozov // Izvestiya Vysshikh Uchebnykh Zavedenii. Power engineering. - Minsk, 1988. - No 1. - P. 123-125.

Paper sent to revision: 25.07.2017.

Paper ready for publication: 03.09.2017. 